\section{OPEN ACCESS}

Edited by:

Rene Duquesnoy,

University of Pittsburgh, United States

Reviewed by:

Frans H. J. Claas,

Leiden University, Netherlands

Lloyd Joseph Andrew D'Orsogna,

Fiona Stanley Hospital, Australia

Lorita Maria Rebellato,

The Brody School of Medicine at East

Carolina University, United States

*Correspondence:

Stanislaw M. Stepkowski

stanislaw.stepkowski@utoledo.edu

Robert C. Green II greenr@bgsu.edu

Specialty section:

This article was submitted to Alloimmunity and Transplantation,

a section of the journal

Frontiers in Immunology

Received: 06 July 2020 Accepted: 02 October 2020

Published: 29 October 2020

Citation:

Bekbolsynov D, Mierzejewska B, Borucka J, Liwski RS,

Greenshields AL, Breidenbach J,

Gehring B, Leonard-Murali S,

Khuder SA, Rees M, Green RC II and

Stepkowski SM (2020) Low

Hydrophobic Mismatch Scores

Calculated for HLA-A/B/DR/DQ LOC Improve Kidney Allograft Survival.

Front. Immunol. 11:580752. doi: 10.3389/fimmu.2020.580752

\title{
Low Hydrophobic Mismatch Scores Calculated for HLA-A/B/DR/DQ Loci Improve Kidney Allograft Survival
}

Dulat Bekbolsynov' ${ }^{1}$, Beata Mierzejewska ${ }^{1}$, Jadwiga Borucka ${ }^{2}$, Robert S. Liwski ${ }^{3}$, Anna L. Greenshields ${ }^{3}$, Joshua Breidenbach ${ }^{1}$, Bradley Gehring ${ }^{1}$, Shravan Leonard-Murali ${ }^{4}$, Sadik A. Khuder ${ }^{5}$, Michael Rees ${ }^{1,6,7}$, Robert C. Green $I^{8^{*}}$ and Stanislaw M. Stepkowski ${ }^{1 *}$

${ }^{1}$ Department of Medical Microbiology and Immunology, University of Toledo, Toledo, OH, United States, 2 Parexel International, Warsaw, Poland, ${ }^{3}$ Department of Pathology, Dalhousie University, Halifax, NS, Canada, ${ }^{4}$ Department of Surgery, Henry Ford Hospital, Detroit, MI, United States, ${ }^{5}$ Department of Medicine and Public Health, University of Toledo, Toledo, OH, United States, ${ }^{6}$ Department of Urology, University of Toledo College of Medicine, Toledo, OH, United States, ${ }^{7}$ The Alliance for Paired Donation, Maumee, $\mathrm{OH}$, United States, ${ }^{8}$ Department of Computer Science, Bowling Green State University, Bowling Green, OH, United States

We evaluated the impact of human leukocyte antigen (HLA) disparity (immunogenicity; IM) on long-term kidney allograft survival. The IM was quantified based on physicochemical properties of the polymorphic linear donor/recipient HLA amino acids (the Cambridge algorithm) as a hydrophobic, electrostatic, amino acid mismatch scores (HMSWAMSIEMS) or eplet mismatch (EpMM) load. High-resolution HLA-A/B/DRB1/DQB1 types were imputed to calculate HMS for primary/re-transplant recipients of deceased donor transplants. The multiple Cox regression showed the association of HMS with graft survival and other confounders. The HMS integer 0-10 scale showed the most survival benefit between HMS 0 and 3. The Kaplan-Meier analysis showed that: the HMS=0 group had 18.1-year median graft survival, a 5-year benefit over HMS $>0$ group; HMS $\leq 3.0$ had 16.7-year graft survival, a 3.8-year better than HMS >3.0 group; and, HMS $\leq 7.8$ had 14.3-year grafts survival, a 1.8-year improvement over HMS>7.8 group. Stratification based on EMS, AMS or EpMM produced similar results. Additionally, the importance of HLA-DR with/without -DQ IM for graft survival was shown. In our simulation of 1,000 random donor/recipient pairs, 75\% with $\mathrm{HMS}>3.0$ were re-matched into $\mathrm{HMS} \leq 3.0$ and the remaining $25 \%$ into $H M S \geq 7.8$ : after re-matching, the 13.5 years graft survival would increase to 16.3 years. This approach matches donors to recipients with low/medium IM donors thus preventing transplants with high IM donors.

Keywords: kidney transplantation, kidney allocation, transplant survival, human leukocyte antigen (HLA), HLA mismatch, immunogenicity

\section{INTRODUCTION}

The impact of the human leukocyte antigen (HLA)-A/B/DR mismatch (MM) on kidney allograft survival has been a subject of extensive research (1-4). While some researchers argue that the role of HLA matching is exaggerated (5), the consensus is that HLA compatibility between a donor and a recipient (HLA immunogenicity) affects long-term kidney outcomes (6). Fully matched (0-HLA-A/ 
B/DR MM) transplants consistently demonstrated the best kidney allograft survival, and fully mismatched (6-HLA-A/B/ DR MM) transplant had the worst outcomes, whereas the remaining 1-, 2-, 3-, 4-, and 5-HLA MM groups had incrementally distributed survival between 0- and 6-HLA MM cohorts $(1,2)$. The graft half-life for kidney transplant from deceased donors was reported as 9.3 years in 2005 and 9.9 years in 2010 (7). Kaplan-Meier and Cox models showed that HLA disparity and multiple other factors may affect kidney allograft survivals (8).

In the U.S., most of ABO-compatible kidney transplants are performed without HLA matching, with the exception for few special programs $(5,9,10)$. Between 2012 and 2016, only $16.4 \%$ of patients received "well-matched" deceased donor kidney transplants at $0-, 1$ - or 2-HLA MM. The remaining $83.6 \%$ of kidney transplants had 3-, 4-, 5-, and 6-HLA MM and therefore should be considered as poorly matched (11). Existing government programs prioritize 0-HLA MM kidney transplants for sensitized patients (12). In particular, the 0MM benefits patients with HLA-A/-B/-DR matched donors who have a panel reactive antibody (PRA) $>20 \%$. From December 2014, the kidney allocation system (KAS) targets patients with a PRA $\geq 98 \%$ and patients waiting $\geq 10$ years on dialysis. The remaining procured kidneys are allocated according to UNOS policies that rarely grant points for HLA matches. Indeed, it is logistically impossible to match HLA for every transplant, and thus less than $10 \%$ of patients receive 0 -HLA MM kidney transplants every year (13).

Not all HLA mismatches are equal, however. The idea of weakly immunogenic HLA mismatches has been used for 30 years in the Acceptable Mismatch program, benefiting over 1,000 very highly sensitized patients (14). Increasing availability of high-resolution HLA typing encourages analysis of molecular disparities in HLA for quantifying HLA immunogenicity beyond antigenic mismatches. One of resulting concepts was HLAMatchmaker for finding immunogenic HLA eplets (15). Eplets represent polymorphic HLA amino acids recognized by $\mathrm{T}$ and B cells (15). Alternatively, the Cambridge algorithm is based on the physicochemical properties of polymorphic HLA amino acid residues $(16,17)$. This algorithm quantifies HLA disparities based on its hydrophobic (HMS), electrostatic (EMS) and amino acid (AMS) properties. These physicochemical-based scores correlated with eplet MM load numbers, as well as the quality of alloantibody response and kidney allograft rejection $(18,19)$, or graft-versus-host disease (GVHD)-free survival after bone marrow transplantation (20). Conceptually, an association was recently shown between donor three-dimensional EMS value and the generation of specific antibody response (21). While other algorithms for HLA IM quantitation exist, the practical and clinically-relevant IM calculation needs to be developed (22).

With decreasing costs and shortening testing times, the 4digit high-resolution HLA typing is an upcoming revolution in transplantation. Recently published rapid testing techniques may soon make it feasible for deceased donor transplants (23). Implementing 4-digit HLA typing has a potential to better avoid unacceptable antigens for sensitized patients. Herein, we explored an additional power of 4-digit HLA typing to provide a benefit of weakly immunogenic transplants and thus improve the long-term graft survival for all patients.

We examined the potential of 4-digit HLAs for finding weakly immunogenic donors. As opposed to the traditional seven integer 0-6-HLA mismatch (MM) system, a continuous physicochemical IM scale based on 4-digit HLA types in HLA$\mathrm{A} / \mathrm{B} / \mathrm{DRB} 1 / \mathrm{DQB} 1$ loci converts antigen mismatch categories into numerical values on a continuous scale. We propose that a continuous IM scale offers greater freedom in finding weakly immunogenic transplants, ultimately allowing improvement of kidney allograft survival. Using the Scientific Registry of Transplant Recipients (SRTR) data, we calculated the HMS, EMS, and AMS by the Cambridge algorithm as well as eplet MM (EpMM) by the HLAMatchmaker for class I (HLA-A/B) and class II (HLA-DRB1/DQB1) HLAs to correlate their values with the long-term kidney allograft survival. We used the Cox regression model to confirm an association between HMS, EMS, AMS, EpMM scores, graft survival, and multiple covariates. Furthermore, we created a simulated allocation model to check whether low/medium immunogenic ABO-compatible donors may be identified for all recipients in comparison to a small fraction of low IM donors identified by an inflexible 0-6 HLA MM system.

\section{MATERIALS AND METHODS}

\section{Patient Population}

The Scientific Registry of Transplant Recipients (SRTR) database was the source of transplant records between 01/01/2000 and 09/04/ 2016 (Figure 1). Out of all 132,515 recipients of deceased donors, high-resolution HLAs were imputed for 78,864 donor/recipient pairs based on available race and HLA-A/B/DR 2-digit antigens; this cohort included 29,852 pairs with HLA-A/B/DRB1/DQB1 types available. The 78,864-cohort had 65,615 patients with primary and 13,249 patients with repeat transplants.

Disclaimer. The SRTR data include all donors, wait-list candidates, and transplant recipients submitted by the members of the Organ Procurement and Transplantation Network (OPTN). The Health Resources and Services Administration (HRSA) is the U.S. Department of Health and Human Services providing an oversight to the activities of the OPTN and SRTR contractors.

\section{Imputation of High-Resolution HLA Types and Calculation of Immunogenicity}

The HaploStats algorithm of the NMDP bioinformatics group was used to impute high-resolution HLA-A/B/DRB1/DQB1 to assign the possible corresponding high-resolution HLA types $(24,25)$. The frequency of HLA haplotypes was calculated based on the distribution of race in the 2011 NMDP full database (26): the most frequent high-resolution haplotype was assigned to the individual. The high likelihood of imputed of HLA alleles was recently demonstrated on a 5,000 cohort of patients in the U.K, 


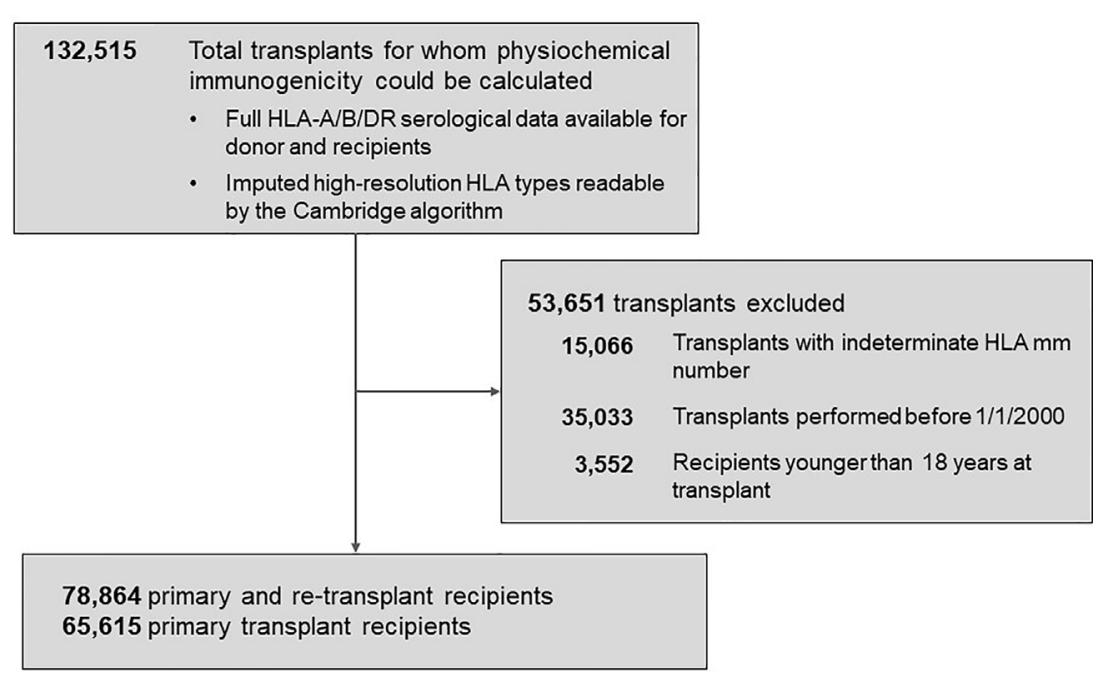

FIGURE 1 | Background characteristics of patient groups represented in the study. The total population of 78,864 patients included 65,615 recipients with primary kidney transplants and 13,249 with re-transplants. Further details are presented in Materials and Methods.

namely $91 \%$ for HLA-A, $88 \%$ for $-\mathrm{B}, 67 \%$ for $-\mathrm{DR}$, and $95 \%$ for -DQ, producing an average of $85 \%$ (27).

The physicochemical IM for each donor-recipient pair was calculated using the Cambridge algorithm, which assigns a score to each amino acid mismatch between donor and recipient HLA $(16,17)$. HLA-A/B/DRB1 or HLA-A/B/DR/DQB1 was used to quantitate the hydrophobic (HMS), electrostatic (EMS), and amino acid (AMS) mismatch scores: IM scores for each transplant were calculated as averages for class I (HLA-A/B) and class II (DR \pm DQ) loci. That way, $50 \%$ of the IM score was contributed by DR alone or DR/DQ combination, as was suggested before (28).

In addition, eplet mismatch (EpMM) scores were calculated in version 3.1 of HLAMatchmaker. Sums of antibody-verified eplet loads in HLA-DRB1 and HLA-A/B loci were used as EpMM values in the sub-cohort of 47,241 patients.

To verify the accuracy of high-resolution HLA types imputation, a cohort of transplant candidates from Queen Elizabeth II Health Sciences Centre, Halifax, Canada ( $\mathrm{n}=$ 1,095) typed to 2-field, P-group resolution at HLA-A/B/C/ DRB1/DQB1 loci using a combination of sequence based typing and extended region SSO methods was used as a reference. Imputed and known high-resolution HLA types and immunogenicity values calculated based on them were then statistically evaluated.

\section{Statistical Analyses}

Graft loss for death-censored graft survival analyses was defined as reported graft failure, resumption of dialysis, re-transplant, or listing for re-transplant. Deaths with functioning graft were censored. Graft half-lives or median survival (time points where $50 \%$ of transplants survived) was established using Kaplan-Meier estimates. When follow-up time was shorter than the graft half-life, a linear survival curve was projected starting from day 4,000 post-transplant.
Four immunogenicity cutoffs were applied to define immunological risk categories that were tested in KaplanMeier survival estimates (see Table 3). Within the HMS scale, the "perfect allelic match" was $\mathrm{HMS}=0$, the "predictive threshold" was HMS $=1.8$ [established by maximally selected rank statistics method, (29)], the "weakly immunogenic" threshold was $\mathrm{HMS}=3.0$ (equal to average HMS in patients with 0-/1-HLA MM) and the "median" threshold was HMS=7.8. Analogous thresholds were defined individually for EMS, AMS and EpMM scales. The same approach was used for HLA MM scale.

Multiple Cox regression models analyzed the association of graft loss risk with immunogenicity and other confounders (Table 1). All data analyses were performed in SAS 9.4 (SAS Institute Inc., Cary, NC) and R (R Foundation for Statistical Computing, Vienna, Austria) programs.

\section{Building the Proportional Hazards Model}

A semi-parametric multiple Cox proportional hazards model estimated the death-censored graft failure as a function of HLA immunogenicity and other confounders. Univariate regression models identified 16 confounders that were significantly associated with death-censored graft survival (Table 1), using a previously described approach (30). With the exception for pretransplant dialysis and recipient education level, all confounders had less than $10 \%$ of missing values; records with missing values were excluded.

All selected variables were tested for the proportional hazard assumption using ASSESS statement of PHREG procedure in SAS. This statement simulated 1,000 possible components $v s$. follow-up time plots $(31,32)$. The Kolmogorov-type supremum test was performed to establish the deviation from the simulated plots of score process components and time, indicating a possible violation of proportionality assumption. Additional verification 
TABLE 1 | Baseline characteristics of patients included in the study ( $\mathrm{n}=$ 78,864).

\begin{tabular}{|c|c|c|}
\hline Variable & $\mathbf{n}$ & $\%$ \\
\hline \multicolumn{3}{|l|}{ Donor age } \\
\hline Less than 18 & 8,399 & 10.7 \\
\hline 18 to 34 & 23,256 & 29.5 \\
\hline 35 to 49 & 24,073 & 30.5 \\
\hline 50 to 64 & 20,278 & 25.7 \\
\hline 65 and over & 2,858 & 3.6 \\
\hline \multicolumn{3}{|l|}{ Recipient age } \\
\hline 18 to 34 & 9,275 & 11.8 \\
\hline 35 to 49 & 22,053 & 27.9 \\
\hline 50 to 64 & 33,348 & 42.3 \\
\hline 65 and over & 14,188 & 18.0 \\
\hline \multicolumn{3}{|l|}{ Donor gender } \\
\hline Male & 46,442 & 58.9 \\
\hline Female & 32,422 & 41.1 \\
\hline \multicolumn{3}{|l|}{ Transplant era } \\
\hline 2000-2004 & 18,121 & 23.0 \\
\hline 2005-2016 & 60,743 & 77.0 \\
\hline \multicolumn{3}{|l|}{ Induction } \\
\hline Yes & 61,523 & 78.0 \\
\hline No & 16,587 & 21.0 \\
\hline Unknown & 754 & 1.0 \\
\hline \multicolumn{3}{|l|}{ Maintenance immunosuppression* } \\
\hline TAC/PRED & 2,556 & 3.2 \\
\hline CsAMMMF/PRED & 5,497 & 7.0 \\
\hline TAC/MMF/PRED & 57,851 & 73.3 \\
\hline Other & 316 & 11.9 \\
\hline Multiple regimens & 9,359 & 0.4 \\
\hline Unknown & 3,285 & 4.2 \\
\hline \multicolumn{3}{|l|}{ Recipient race } \\
\hline White & 36,435 & 46.2 \\
\hline African American & 27,618 & 35.0 \\
\hline Asian & 4,131 & 5.2 \\
\hline Hispanic/Latino & 9,721 & 12.3 \\
\hline Other & 959 & 1.3 \\
\hline \multicolumn{3}{|l|}{ Donor race } \\
\hline White & 62,376 & 79.1 \\
\hline African American & 13,064 & 16.6 \\
\hline Asian & 1,891 & 2.4 \\
\hline Hispanic/Latino & 976 & 1.2 \\
\hline Other & 557 & 0.7 \\
\hline \multicolumn{3}{|l|}{ Recipient education } \\
\hline None, grade school or high school & 37,073 & 47.0 \\
\hline Attended college, bachelors or post-college & 32,207 & 40.8 \\
\hline Unknown & 9,584 & 12.2 \\
\hline \multicolumn{3}{|l|}{ Recipient primary source of payment } \\
\hline Private insurance & 31,976 & 40.5 \\
\hline Public insurance & 34,302 & 43.5 \\
\hline Other & 12,370 & 15.7 \\
\hline Unknown & 216 & 0.3 \\
\hline \multicolumn{3}{|l|}{ Cold ischemia time } \\
\hline Less than 24 hours & 58,092 & 73.7 \\
\hline 24 hours or more & 15,508 & 19.7 \\
\hline Unknown & 5,264 & 6.6 \\
\hline \multicolumn{3}{|l|}{ Waiting time } \\
\hline Less than 1 year & 25,497 & 32.3 \\
\hline 1 to 3 years & 29,350 & 37.2 \\
\hline 3 to 5 years & 15,980 & 20.3 \\
\hline Over 5 years & 8,037 & 10.2 \\
\hline \multicolumn{3}{|l|}{ Candidate $\mathrm{BMI}>30$} \\
\hline Yes & 27,745 & 35.2 \\
\hline No & 29,510 & 62.8 \\
\hline Unknown & 1,609 & 2.0 \\
\hline
\end{tabular}

(Continued)
TABLE 1 | Continued

\begin{tabular}{lcc}
\hline Variable & $\mathbf{n}$ & $\%$ \\
\hline Candidate pre-transplant diabetes & & \\
No & 51,811 & 65.7 \\
Type I & 2,482 & 3.2 \\
Type II & 16,069 & 20.4 \\
Other & 8,329 & 0.4 \\
Unknown & 173 & 10.3 \\
Pre-transplant dialysis & & \\
No dialysis & 15,219 & 19.3 \\
Hemodialysis & 45,586 & 57.8 \\
Peritoneal Dialysis & 8,345 & 10.6 \\
Continuous Venous/Venous & 739 & 0.9 \\
Hemofiltration & & \\
Unknown & 8,975 & 11.4 \\
\hline${ }^{*}$ PRED indicates the use of steroids or steroid-free regimen. & & \\
\end{tabular}

was performed using Schoenfeld residuals and log-negative log plots for each variable (not shown). Variables violating the proportional hazard assumption were included in the Cox model with time log transformation (33).

\section{Simulated Allocation Model}

We have designed a simulated allocation algorithm to test the maximum number of donors that could be found for recipients within a designated HMS threshold. When the HMS was greater than the designated threshold, the pair was broken and simulation re-run with other broken pairs until the number of pairs under the designated HMS threshold stopped growing. Thus, a maximum number of low IM pairs within a given population was established. Four input populations $(\mathrm{n}=10, \mathrm{n}=$ $100, \mathrm{n}=1,000$, or $\mathrm{n}=5,000$ ) and five IM thresholds (HLA MM $\leq$ 1 or HMS $=0$, HMS $\leq 3.0$, HMS $\leq 5.5$ or HMS $\leq 7.8$ ) were tested. This procedure was repeated 10 times always with new pairs for all except 5,000 pairs, which was run only once as re-matching was prohibitively lengthy.

\section{RESULTS}

\section{Patient Demographics and 4-Digit HLA Imputation}

A total of 78,864 kidney primary and re-transplants from the SRTR were included into cohort. Baseline characteristics of the cohort are presented in Table 1. There were 30,526 (38.7\%) female recipients and 48,338 (61.3\%) male recipients with mean age at transplant of 51.9 years; 13,553 patients died with a functioning kidney. Among donors, 32,422 (41.1\%) were female 46,442 (58.9\%) were male; their mean age was 38.4 years. Recipients were represented by mostly non-Hispanic whites (46.2\%), while $35.0 \%$ were black. Recipients who had at least a high school education or less represented most of patients (47.0\%), whereas $40.8 \%$ of patients attended college. Most patients (80.7\%) were on hemodialysis prior transplantation.

To assure the accuracy of our analyses, the same imputation from 2-digit to 4-digit HLA-A/B/DRB1/DQB1 types was performed for the individuals typed at Queen Elizabeth II Health Sciences 
Centre with high resolution at HLA-A/B/DRB1/DQB1 loci $(\mathrm{n}=$ 1,095). The imputed high-resolution was $96 \%$ identical in HLA-A locus, $92 \%$ in -B locus, $73 \%$ in -DRB1 locus, and $85 \%$ in -DQB1 locus (Table 2). In addition, we used the above individuals in an experiment to test the impact of imputed HLAs vs. real HLAs on the accuracy of IM calculation in our analysis: 547 presumed donors and 547 presumed recipients were used to calculate HMS/EMS/ AMS values. In paired t-test, there was no difference between HMS $(\mathrm{p}=0.31)$, EMS $(\mathrm{p}=0.36)$, and AMS $(\mathrm{p}=0.37)$ values of imputed vs. real 4-digit HLA types. Thus, the HLA imputation had little, if any, impact on this proof-of-concept results (Table 2).

\section{Association of HLA Mismatch Levels With HMS Immunogenicity Scores}

Currently, the HLA IM between a donor and a recipient is defined at 2-digit level as 0-6 HLA-A/B/DR MM. We investigated the association of kidney allograft survival where HLA IM was defined at 4-digit level by the Cambridge algorithm $(16,17)$. The HMS, EMS, and AMS MM scores strongly correlated based on intra-class correlation coefficient of $82.5 \%$ (HMS/EMS linear regression at $\mathrm{R}^{2}=0.90$ ), as previously reported (16). When HMS, EMS, and AMS values were plotted on the 0to 6-HLA MM scale, the 1- and 2-HLA MM groups had the lowest whereas the 5- and 6-HLA MM groups had the highest average HMS values (Figures 2A-C). However, while the low IM scores were found predominantly among 0-, 1-, and 2-HLA MM pairs, they were also present in 3-, 4-, 5- and 6-HLA MM groups. The same was true for highly IM transplants, high HMS values were found in the 1- to 6-HLA MM groups. Similar HMS distribution patterns were in the primary and re-transplant cohorts (not shown).

\section{Multiple Cox Regression Analysis}

A semi-parametric multiple Cox proportional hazard method estimated the association of HMS values with other confounders on the risk for graft failure. Selected confounders showed a significant association with the death-censored graft survival in univariate Cox regression models (Supplemental Table 1). Those variables were then tested for the proportional hazard assumption using ASSESS statement of PHREG procedure in SAS $(31,32)$, and variables violating the proportional hazard assumption were included with time log transformation (33). All HLA MM, and HMS values were significantly associated with graft loss. In conclusion, the HMS-measured IM had significant association with the death-censored graft survival in multiple regression models (Supplemental Table 2). In addition, immunosuppression, induction therapy, PRA levels, and cold ischemia time were associated with the risk of graft loss $(\mathrm{p}<$ 0.001; Supplemental Table 2).

\section{Low Immunogenicity Scores in HLA-A/B/ DR Correlate With Better Kidney Allograft Survival}

Overall, our 78,864 cohort had a 10-year kidney transplant survival of $62.3 \%$ with half-life of 13.5 years (Figure 3A). Traditional 0 - to 6HLA MM plots showed an incremental decrease in death-censored graft survival (Figure 3B): 0-MM had 71\% 10-year survival with half-life of 15.5 years, while 6-MM had only 57\% 10-year survival with graft half-life of 11.8 years (Figures 3A, B; Table 3). The same pattern was for primary/re-transplant or just primary (not shown) transplants, as was previously described $(1,2)$.

Our goal was to select an HMS threshold with the largest number of patients producing graft survivals as in a 0-/1-HLAA/B/DR MM cohort. In particular, we stratified transplant recipients into four immunological risk categories based on HMS (EMS, AMS, or EpMM) thresholds and analyzed their graft failure rates in Kaplan-Meier survival estimates. The HMSbased thresholds were HMS=0 (perfect allelic match), HMS=1.8 (predictive threshold), HMS=3.0 (low IM threshold; equal to mean HMS in 0-/1-HLA MM), and HMS=7.8 (median HMS threshold). EMS, AMS, and EpMM-based thresholds were defined similarly and their corresponding values (Table 3).

In detailed analysis, the perfect allelic match HMS $=0$ selected 4,899 patients with graft half-life of 18.1 years, gaining 5.0 years over 13.1 years for the HMS $>0$ group ( $<<0.001$; Figure $3 C$; Table 3). The predictive threshold based on the maximallyselected statistics (29) calculated an $\mathrm{HMS}=1.8$ as low-risk predictor for the graft loss ( $\mathrm{p}<0.001$; Figure 3D). The HMS $\leq$ 1.8 group had 7,846 patients with 16.6-year graft half-life, a 3.6year improvement over 13.0 years in the HMS $>1.8$ group $(p>$ 0.001 ; Figure 3D; Table 3). In another analysis, with the $0-10$ HMS integer scale, the most gain for the graft survival was observed in transplants with HMS up to 3.0. This HMS $\leq 3.0$ threshold included 11,483 transplants with 16.7-year graft survivals, a 3.8-year gain over 12.9-year in the HMS $>3.0$ group $(\mathrm{p}<0.001$; Figure 3E; Table 3). Finally, the median HMS $\leq 7.8$

TABLE 2 | Identity between the known and imputed high-resolution types in the 1,095 patients from Haifax (upper part) and similarity between physicochemical immunogenicity levels calculated based on them (lower part).

\begin{tabular}{|c|c|c|c|c|c|c|}
\hline HLA Locus & \multicolumn{2}{|c|}{ A } & B & DR & \multicolumn{2}{|c|}{$\mathbf{D Q}$} \\
\hline$\%$ of Identical typing & \multicolumn{2}{|c|}{96.1} & 92.7 & 73.8 & \multicolumn{2}{|c|}{85.3} \\
\hline \multicolumn{7}{|c|}{ Immunogenicity calculated based on real or imputed high-resolution HLA types } \\
\hline \multirow[t]{2}{*}{ Immunogenicity measure } & \multicolumn{2}{|c|}{ HMS } & \multicolumn{2}{|c|}{ EMS } & \multicolumn{2}{|c|}{ AMS } \\
\hline & Real & Imputed & Real & Imputed & Real & Imputed \\
\hline Mean & 12.04 & 11.91 & 12.22 & 12.11 & 8.23 & 8.17 \\
\hline Paired t-test p-value & \multicolumn{2}{|c|}{0.316} & \multicolumn{2}{|c|}{0.362} & \multicolumn{2}{|c|}{0.369} \\
\hline
\end{tabular}


TABLE 3 | Graft half-lives based on death-censored graft survival analyses using the different immunogenicity scales in the primary/re-transplants cohort. Immunogenicity was calculated based on HLA-A/B/DR loci. Graft half-life values over 16.1 years were imputed as described in Materials and Methods section.

\begin{tabular}{|c|c|c|c|c|}
\hline \multirow[t]{2}{*}{ Immunogenicity Cutoff value } & \multicolumn{2}{|c|}{ Graft Half-Life, years $\left(95 \% \mathrm{Cl}^{\star}\right)$} & \multicolumn{2}{|c|}{$\mathbf{n}$} \\
\hline & Below Cutoff & Above Cutoff & Below Cutoff & Above Cutoff \\
\hline \multicolumn{5}{|l|}{ 0-6 MM scale M $^{\star \star}$} \\
\hline Antigenic Match (MM = 0) & $15.5(15.1-\mathrm{NA})$ & $13.4(13.1-13.7)$ & 5,971 & 72,208 \\
\hline Predictive Threshold (MM = 3) & $15.1(14.6-15.6)$ & $13.0(12.7-13.3)$ & 19,826 & 58,353 \\
\hline Weakly Immunogenic (MM $\leq 1)$ & $16.1(15.1-\mathrm{NA})$ & $13.3(13.0-13.6)$ & 6,723 & 71,456 \\
\hline Median (HLA MM = 4) & $14.4(14.1-14.9)$ & $12.9(12.3-13.2)$ & 39,853 & 38,326 \\
\hline \multicolumn{5}{|l|}{ HLA-A/B/DR HMS scale } \\
\hline Allelic Match $(\mathrm{HMS}=0)$ & $18.1(15.1-\mathrm{NA})$ & $13.4(13.1-13.7)$ & 4,899 & 73,884 \\
\hline Predictive Threshold (HMS = 1.8) & $17.0(15.1-\mathrm{NA})$ & $13.3(13.3-13.0)$ & 7,846 & 70,945 \\
\hline Weakly Immunogenic (HMS = 3.0) & 16.7 (15.7-NA) & $12.9(9.8-13.8)$ & 11,483 & 67,381 \\
\hline Median $(\mathrm{HMS} \leq 7.8)$ & $14.3(14.0-14.6)$ & $12.5(12.2-13.0)$ & 39,439 & 39,425 \\
\hline \multicolumn{5}{|l|}{ HLA-A/B/DR EMS scale } \\
\hline Allelic Match $(E M S=0)$ & $17.1(15.1-\mathrm{NA})$ & $13.4(13.1-13.7)$ & 4,701 & 74,163 \\
\hline Predictive Threshold (EMS = 1.8) & $16.2(15.1-\mathrm{NA})$ & $12.8(12.4-13.2)$ & 7,858 & 71,006 \\
\hline Weakly Immunogenic (EMS = 3.6) & $16.7(15.1-\mathrm{NA})$ & $13.2(12.9-13.6)$ & 14,165 & 64,699 \\
\hline Median $(E M S=7.9)$ & $14.4(14.2-15.0)$ & $12.8(12.4-13.1)$ & 39,226 & 39,638 \\
\hline \multicolumn{5}{|l|}{ HLA-A/B/DR AMS scale } \\
\hline Allelic Match (AMS = 0) & $17.1(15.1-\mathrm{NA})$ & $13.4(13.1-13.7)$ & 4,899 & 73,965 \\
\hline Predictive Threshold (AMS = 2.5) & $15.6(15.1-\mathrm{NA})$ & $13.2(12.9-13.5)$ & 9,782 & 69,082 \\
\hline Weakly Immunogenic (AMS = 2.3) & $15.3(15.0-N A)$ & $13.1(12.9-13.4)$ & 9,712 & 69,647 \\
\hline Median (AMS = 5.8) & $14.4(14.1-14.8)$ & $12.9(12.4-13.2)$ & 25,841 & 53,023 \\
\hline \multicolumn{5}{|c|}{ HLA-A/B/DR Antibody-Verified Eplet load scale } \\
\hline Allelic Match $(E p M M=0)$ & $22.6(\mathrm{NA}-\mathrm{NA})$ & $15.3(15.0-15.6)$ & 1,584 & 45,657 \\
\hline Predictive Threshold (EpMM = 2) & 17.5 (NA-NA) & $15.1(14.9-15.6)$ & 6,335 & 40,906 \\
\hline Weakly Immunogenic (EpMM = 1.7) & 18.3 (NA-NA) & $15.1(14.9-15.6)$ & 5,419 & 41,882 \\
\hline Median (EpMM = 5.5) & $15.2(14.4-16.1)$ & $13.5(13.2-13.8)$ & 21,170 & 20,071 \\
\hline
\end{tabular}

*NA in confidence intervals indicates the upper survival limit was too far in the future to be computed in PROC LIFETEST procedure.

${ }^{* *}$ The total numbers of patients in HLA MM categories is 78,179 , reflecting records with blank HLA MM variable values.

cohort achieved 14.3-year survival, a 1.8-year better than 12.5 years for HMS $>7.8$ cohort $(\mathrm{p}<0.001$; Figure 3F; Table 3). Notably, the transplants with HMS $\leq 1.8$ and HMS $\leq 3.0$ had graft survival better or equal to that in the 0-/1-HLA MM cohort (Figure 4); however, the number of patients in HMS $\leq 1.8$ and HMS $\leq 3.0$ was significantly higher than in the 0 -/1-HLA MM cohort (Table 3). The EMS, AMS, and EpMM values showed similar patterns with their own specific thresholds; EpMM evaluation displayed comparable results (Table 3 ).

\section{The Dominant Role of -DR With -DQ HMS Values in Kidney Allograft Survival}

Since donor class II -DR and -DQ mismatches proved to be very important in kidney allograft survivals (19,34-36), we examined their impact at the same HMS thresholds. The 29,852 HLA-A/B/
DR/DQ cohort showed that recipients matched at -DR alone had grafts surviving 15.3 years while those matched at -DQ alone 15.0 years (Table 4). The HMS $=0$ group matched at HLA-A/B/DR exhibited an extra benefit from an additional matching at -DQ with 18.4 years $v s$. 15.6-year survivals with mismatch at -DQ, an almost 3.0-year benefit (Table 4); similar gains were produced by -DQ matching at HMS $\leq 3.0$ and HMS $\leq 7.8$ (Table 4). The importance of -DR over -DQ was shown when the entire cohort was matched $v s$. mismatched at only -DR (Figure 5A) as well as matched $v s$. mismatched at only -DQ (Figure 5B), as the benefit of additional -DQ match in -DR matched transplants was not statistically significant. Similar patterns were also displayed when HMS $\leq 3.0$ was calculated only for -DR or -DQ (Figures 5C, D). However, patients with HMS matched at HLA-A/B/DR had an extra benefit from an additional -DQ match (Table 4,
A

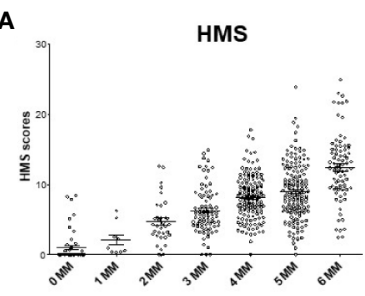

B

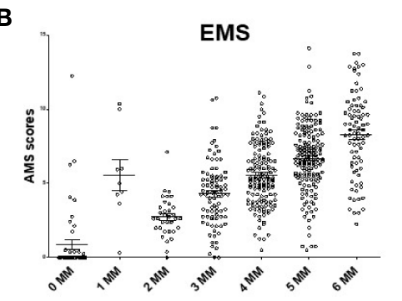

C

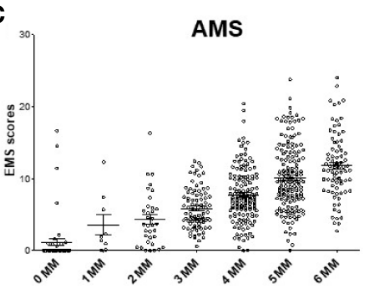

FIGURE 2 | The distribution of physicochemical HMS, EMS, and AMS mismatch (scores across the antigenic 0-6-HLA MM groups. Each dot represents 200 donor-recipient pairs; Panels (A) (HMS), (B) (EMS) and (C) (AMS) has 78,864 recipients of primary or repeat kidney allografts. More details in Materials and Methods. 


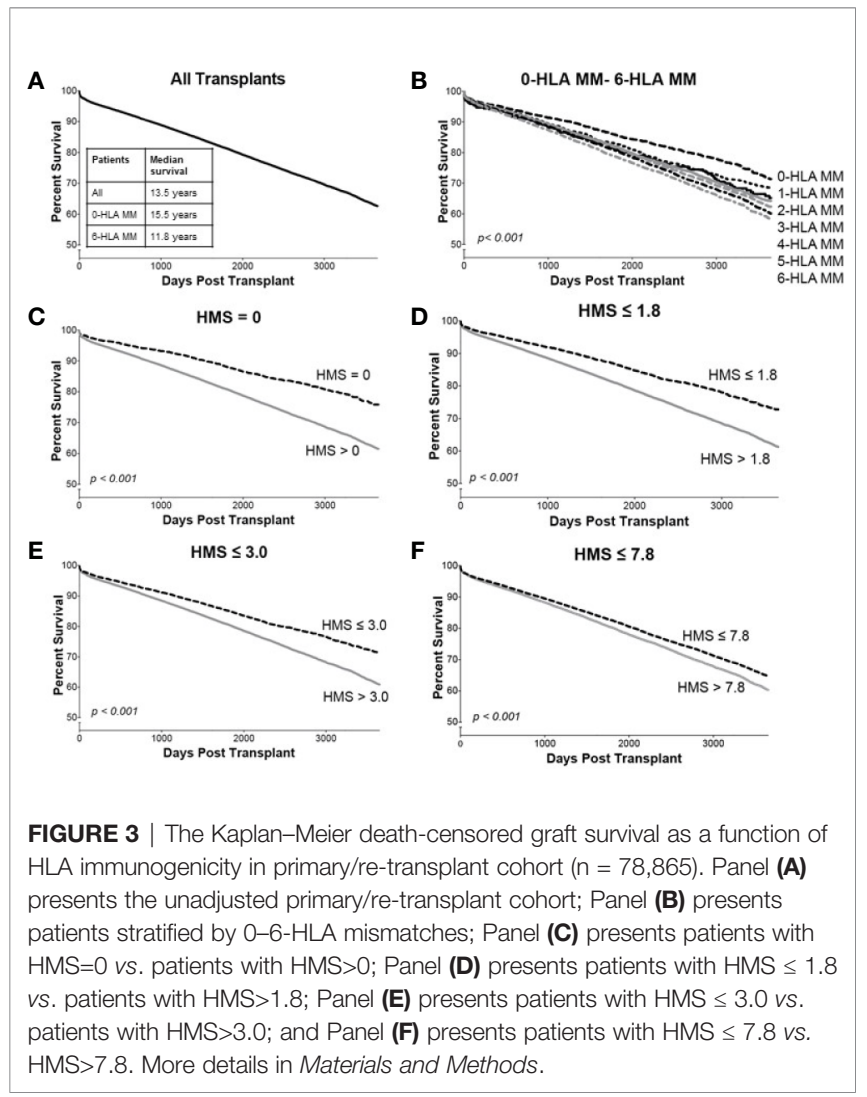

Supplemental Figures 1A-C). This benefit was stronger from -DR matching in transplants already matched by -DQ than vice versa (Supplemental Figures 1D, E). Our conclusion is that the -DRB1 locus plays the dominant role in the HMS values, but additional matching at -DQB1 locus improves an overall graft survival.

\section{Simulated Matching to Generate the Maximum Possible Number of Weakly- Immunogenic Donor/Recipient Pairs}

So far, only $6 \%$ of deceased donor transplants were fully matched (11), while $83.6 \%$ of transplants had more than three HLA-A/B/DR mismatches. The new kidney allocation policy by OPTN (Organ Procurement and Transplantation Network) establishes a 250-mile radius procurement regions for deceased kidney harvesting, assigning in the order of $\approx 1,000$ deceased kidneys per region (37). We tested whether HMS thresholds could reshuffle donors to recipients to maximize those with $\mathrm{HMS} \leq 3.0$, HMS $\leq 5.5$, or HMS $\leq 7.8$ (Table 5). Random donor/recipient pair populations were re-matched to form the maximum possible number of new pairs that would have HMS or HLA MM value within the designated IM threshold. ABO compatibility was or was not enforced in simulated pairs. This approach re-matched up to $85 \%$ of pairs under HMS $\leq 3.0$ in the population of 5,000 input pairs (Table 5). Furthermore, enforcing HMS $\leq 7.8$ threshold produced 95\% matches for 1,000 and $99 \%$ for 5,000 random pairs. Rematched weakly immunogenic donor/recipient pairs had recipient $\mathrm{ABO}$ distribution similar to that in the general population (Supplemental Table 3). Enforcing ABO matching produced slightly lower percentages of donor/recipient pairs that could be re-matched, though that difference was small in $\mathrm{n}=1,000$ and $\mathrm{n}=$ 5,000 trial sizes (Table 5). Remarkably, re-matching of 1,000 pairs to obtain the largest number of 0-/1-HLA MM pairs produced only $12.5 \%$ ( $\pm 0.85 \%)$ matches (Table 5). Thus, a continuous IM scale may find low IM donors for most patients and may exclude all transplants with high IM donors (HMS>7.8).

\section{DISCUSSION}

The main message is that a continuous HLA IM scale finds lowIM donors better than the current HLA mismatch scale. We
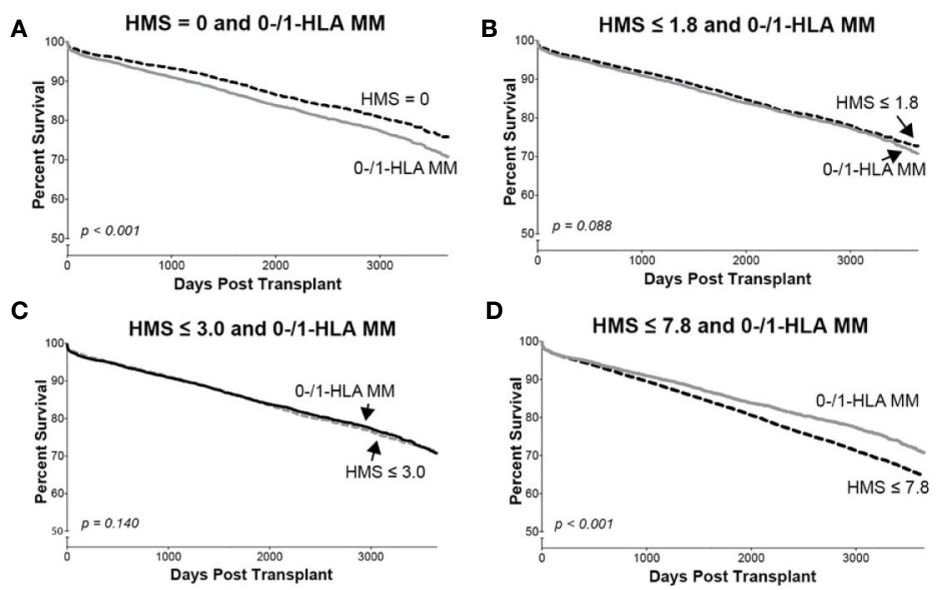

FIGURE 4 | The Kaplan-Meier death-censored graft survival in patients selected by different HMS thresholds compared to patients with combined 0-/1-HLA MM selected from the primary/re-transplant cohort $(n=78,865)$. Patients with $\mathrm{HMS}=0$ (A), patients with $\mathrm{HMS} \leq 1.8$ (B), patients with $\mathrm{HMS} \leq 3.0$ (C), and patients with HMS $\leq 7.8$ (D) were compared to the patients in the same cohort with 0-/1-HLA MM. 
TABLE 4 | HLA-class II immunogenicity and graft survival. Graft half-lives based on death-censored graft survival analyses with 0- to 2-HLA MM scale and the flexible HMS scale in the primary/re-transplants cohort. Immunogenicity was calculated based on HLA- DRB1 and/or HLA-DQB1 loci.

\begin{tabular}{|c|c|c|c|}
\hline HLA-DRB1 or -DQB1 & $\mathbf{N}$ & Graft half-life, years $(95 \% \mathrm{Cl})$ & $95 \% \mathrm{Cl}^{*}$ \\
\hline HLA-DR MM = 0 & 15,014 & 15.3 & 15.1-NA \\
\hline HLA-DQ MM = 0 & 5,775 & 15.0 & 14.3-NA \\
\hline HLA-A/B/DR/DQ & $\mathbf{N}$ & Graft half-life, years (95\% Cl) & $95 \% \mathrm{Cl}^{*}$ \\
\hline $\mathrm{A} / \mathrm{B} / \mathrm{DR} \mathrm{HMS}=0,-\mathrm{DQ} \mathrm{MM}=0$ & 1,864 & 19.2 & NA-NA \\
\hline $\mathrm{A} / \mathrm{B} / \mathrm{DR} \mathrm{HMS}=0,-\mathrm{DQ} \mathrm{MM}=1$ or 2 & 1,068 & 18.1 & 15.1-NA \\
\hline $\mathrm{A} / \mathrm{B} / \mathrm{DR} \mathrm{HMS} \leq 3.0,-\mathrm{DQ} \mathrm{MM}=0$ & 2,773 & 18.4 & 15.0-NA \\
\hline $\mathrm{A} / \mathrm{B} / \mathrm{DR} \mathrm{HMS} \leq 3.0,-\mathrm{DQ} \mathrm{MM}=1$ or 2 & 2,846 & 15.6 & 15.1-NA \\
\hline $\mathrm{A} / \mathrm{B} / \mathrm{DR} \mathrm{HMS} \leq 7.8,-\mathrm{DQ} \mathrm{MM}=0$ & 4,538 & 15.3 & 14.6-NA \\
\hline $\mathrm{A} / \mathrm{B} / \mathrm{DR} \mathrm{HMS} \leq 7.8,-\mathrm{DQ} \mathrm{MM}=1$ or 2 & 11,289 & 13.9 & $13.1-15.1$ \\
\hline Matched by HLA-DRB1 & $\mathbf{N}$ & Graft half-life, years (95\% Cl) & $95 \% \mathrm{Cl}^{*}$ \\
\hline HLA-DR MM = 0, HLA-DQ MM = 0 & 3,883 & 15.3 & 15.0-NA \\
\hline HLA-DR MM = 0, HLA-DQ MM = 2 & 624 & 16.1 & 9.7-NA \\
\hline Matched by HLA-DQB1 & n & Graft half-life, years $(95 \% \mathrm{Cl})$ & $95 \% \mathrm{Cl}^{*}$ \\
\hline HLA-DQ MM = 0, HLA-DR MM = 0 & 3,883 & 15.3 & 15.0-NA \\
\hline HLA-DQ MM = 0, HLA-DR MM = 2 & 457 & 12.7 & 13.9-NA \\
\hline
\end{tabular}

*NA in confidence intervals indicates the upper survival limit was too far in the future to be computed in PROC LIFETEST procedure.

report herein four main findings. First, the IM, calculated for HLA-A/B/DR/DQ of each donor/recipient pair by the Cambridge algorithm (HMS) correlated with long-term kidney allograft survivals. Second, low IM donors were selected on a flexible scale ranging from zero to median HMS values. Third, the HMS $\leq 3.0$ threshold had an excellent 16.7-year graft survival with a 3.8-year benefit over HMS $>3.0$ transplants; the median HMS $\leq 7.8$ threshold had 14.2-year graft survival, a 2.8-year benefit. Fourth, $75 \%$ of 1,000 random ABO-matched patients were then re-matched with HMS $\leq 3.0$ value and the remaining $25 \%$ with the medium HMS 3.0-7.8 value. Overall, such approach in our cohort would increase the graft half-life from 13.5-years to 16.4 years. Thus, the high-resolution HLA typing with the HMS-based matching could revolutionize the selection of donors.
The 2-digit 0-HLA MM group with graft survival of 15.5 years included the "real" 4-digit 0-HLA MM transplants $(\mathrm{HMS}=0$ ) with superior graft survival of 18.1 years. Thus, only precise 4digit HLA matching produced "perfect" matches with superior outcomes, otherwise achieved only by living related donors (38). This was as equally impressive as 16.7-year graft half-life among 11,483 patients with HMS $\leq 3.0$ selected from the 0 - to $6-\mathrm{MM}$ cohort (Table 3). The median HMS $\leq 7.8$ threshold had graft survivals of 14.3 years and that should be the upper limit to avoid high IM transplants (HMS > 7.8). The survival benefit in weakly immunogenic transplants is excellent in recipients of kidneys with full allelic match, but when $\mathrm{HMS}=0$ patients were excluded from HMS $\leq 3.0$ cohort, the graft survival still was significantly higher than in HMS>3.0 cohort (14.6 vs 12.9 years, not shown). While HLA-DR was dominant in contributing to the HMS
A

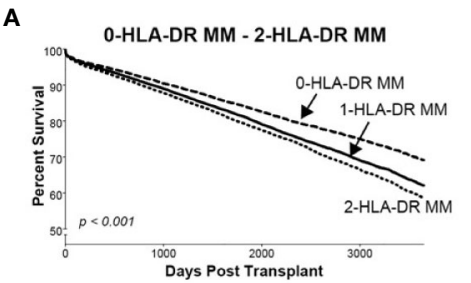

C

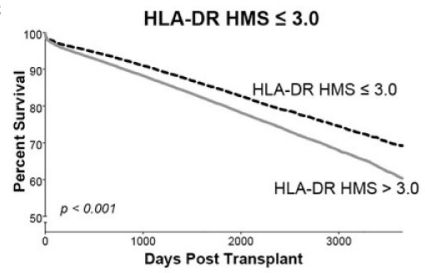

B

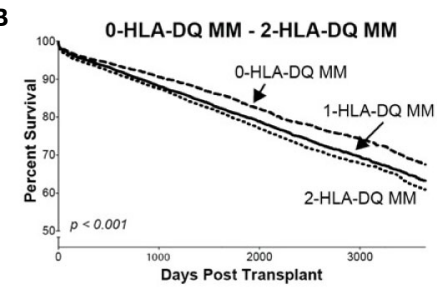

D

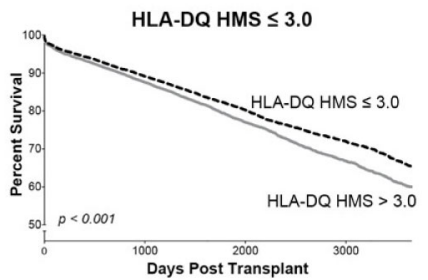

FIGURE 5 | The Kaplan-Meier death-censored graft survival as a function of class II (HLA-DR and -DQ) immunogenicity in primary/re-transplant cohorts ( $\mathrm{n}=$ 78,865). Panels (A, B) present patients stratified by mismatches at 0-, 1- or 2-HLA-DR (A) or -DQ (B) antigens. Transplants from donors with split broad antigens (for example, HLA-DR3 in donor and HLA-DR17 in recipient-this could be either a match or a mismatch) were excluded from the analysis; Panel (C) presents patients stratified by HLA-DR HMS $\leq 3.0$ vs. HLA-DR HMS>3.0, and Panel (D) presents patients stratified by HLA-DQ HMS $\leq 3.0$ vs. HLA-DQ HMS>3.0. 
benefit, matching at -DQ locus further improved allograft survival in patients with low HMS scores (Table 4); the -DQ analysis was limited by $30 \%$ availability of this locus typing in the SRTR database. Overall, we propose that the Cambridge algorithm proffers a versatile way to identify low/medium IM donors independent of 0-6 HLA matching: the best results were produced by HMS matching at the combined HLA-A/B/DR/ DQ loci.

A continuous IM scale is versatile. In the retrospective cohort of 78,864 recipients, there were $11 \%$ more pairs with $\mathrm{HMS} \leq 1.8$ than those with 0-/1-HLA MM. Similarly, there were 35\% more pairs with HMS $\leq 3.0$ than those with 0 -/1-HLA MM. Given the median graft survival in HMS $\leq 1.8$ and $\mathrm{HMS} \leq 3.0$ groups was comparable to that in the 0-/1-HLA MM group (Table 3), it is easy to see that substantially better survival results could be produced if more effort was put in finding weakly immunogenic donors on a continuous IM scale. Exploring this insight, our simulation found that $75 \%$ of 1,000 random pairs could be rematched with low (HMS $\leq 3.0)$ and remaining $25 \%$ with medium (HMS 3.0-7.8) IM donors (Table 5). This would increase an average graft survival by $21 \%$ from 13.5 years to 16.4 years producing extra 224,762 kidney life years in 78,864 patients. While we did take into consideration $\mathrm{ABO}$ compatibility, unacceptable HLA antigens data was not available in the SRTR database. Still, HMS alone and ABO blood groups/HMS rematching simulation provided a proof of concept: a random 10 to 5,000 pool of donor/recipient contain sufficiently diverse HLA haplotypes to accommodate most if not all recipients with weakly immunogenic grafts. We plan to quantify benefits using real-life simulation conditions.

The proportional hazard regression already examined the impact of HLA on graft survival with multiple covariates (2). This multifactorial analysis provided tools for addressing assumptions violations in the Cox model. Proportional hazard distribution is one of the most important prerequisites in a model validation $(39,40)$. Williams and his colleagues did not find substantial deviations from the proportionality in their research cohorts (2). Our data demonstrated significant nonproportionality with most confounders, prompting our use of time log transformation to rectify our model (33). It is possible that the strict definition of proportionality could have contributed to the difference. Despite this disparity, both Cox analyses revealed that immunosuppression and induction therapy had the highest impact $(\mathrm{p}<0.001)$ on the IMdependent graft survival (2). Thus, immunosuppression and induction covariates are affecting the HLA-based matching of donors and recipients.

While imputation is not recommended for clinical decision making (39), it is an invaluable tool for research. We have developed an algorithm for fast imputation of high-resolution HLA types on the HaploStats server (26). The proportion of HLA alleles imputed with high likelihood was shown on 5,000 volunteers with $91 \%$ accuracy for HLA-A, $88 \%$ for -B, $67 \%$ for -DRB1, and 95\% for-DQB1 (an average of 85\%) in previously published research (27). Our analysis of 1,095 individuals produced better results: the imputed/real high-resolution was 96\% precise for HLA-A locus, 92\% for -B locus, 73\% for -DRB1 locus, and $85 \%$ for -DQB1 locus (Table 2). Future clinical application needs bona fide 4-digit HLA typing rather than imputed HLA identification.

The half-life of kidney grafts from deceased donors has slightly improved from 7.4 years in 1994 to 9.8 years in 2008 (11). An independent 20-year study confirmed graft half-life of 9.3 years in 2015 (41). The 2-year improvement can be attributed to better induction and immunosuppression therapy in almost all recipients $(42,43)$. In the U.S., HLA matching is not done because of the logistic complexity of the 0- to 6-HLA scale and the desire to avoid disadvantaging minority patients. The sensitization of patients further complicates selection of donors (44-46). Our approach may help avoid unacceptable HLA alleles when finding low IM donors.

Analysis of donor eplets, based on 4-digit HLAs, became necessary to overcome an increasing number of new HLA alleles (47). In fact, one very immunogenic eplet may be present on multiple HLA molecules requiring their avoidance in donor selection. The low HLA eplet mismatch (EpMM) load has been considered as an extension of 2-digit HLA antigens in improving donor choice, especially for children (48). Indeed, modified

TABLE 5 | Simulation analyses to re-match 10, 100, 1,000 or 5,000 donor/recipeint pairs with $\mathrm{HMS}=0$, HMS $\leq 3.0, \leq 5.5$, or $\leq 7.8$.

\begin{tabular}{|c|c|c|c|c|c|}
\hline Immunogenicity threshold & ABO-matched & \multicolumn{4}{|c|}{ Percent of re-matched pairs } \\
\hline HMS $=0$ & No & 1.00 & 6.90 & 16.10 & 23.53 \\
\hline \multirow[t]{2}{*}{$\mathrm{HMS} \leq \mathbf{3 . 0}$} & No & 15.91 & 44.00 & 72.98 & 84.85 \\
\hline & Yes & 7.00 & 32.60 & 63.61 & 79.07 \\
\hline HMS $\leq 5.5$ & No & 47.00 & 71.40 & 88.00 & 93.20 \\
\hline $\mathrm{HMS} \leq 7.8$ & Yes & 46.00 & 73.60 & 90.32 & 96.26 \\
\hline HLA MM $\leq 1$ & No & 1.00 & 3.20 & 14.49 & $N D^{\star \star}$ \\
\hline
\end{tabular}

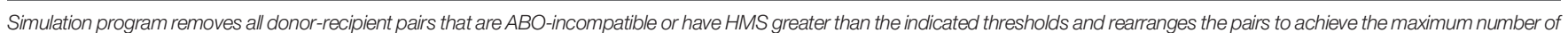
pairs with HMS $=0, \geq 3.0, \geq 5.5$, or $\geq 7.8$, or HLA MM $\leq 1$. The experiment with 1,000 pairs was repeated 10 times, whereas the experiment with 5,000 pairs was repeated once. ${ }^{*} n$ - population of donor/recipients pair size.

${ }^{* *} N D-$ not done. 
Poisson regression showed that the EpMM load was an effective tool in the risk assessment. In other work, EpMM values were correlated with AMS and EMS scores $\left(\mathrm{R}^{2}=0.85-0.96\right)$ in de novo donor-specific antibody (dnDSA) production (19). Furthermore, all three methods (EpMM, EMS, and AMS) correlated with dnDSA following adjustment of variables such as recipient age, immunosuppression, and non-compliance. Overall, the authors suggested to use the easiest method as similar results were produced by each method. We argue about the use of HMS, EMS or AMS values. However, we also recognize the qualitative differences among antibody-verified eplets as more or less immunogenic, as others showed $(47,49,50)$. Thus, we believe that future approach should combine the HMS threshold with antibody-verified EpMM analysis for the best pre-transplant risk assessment.

In summary, we propose to prioritize low IM donors by utilizing HMS-based selection instead of the rigid 0-6-HLA system. Relying on physicochemical properties provides flexibility and may accommodate all or almost all recipients. The application of the HMS system for all deceased donor kidney transplants in United States should significantly increase graft survivals.

\section{DATA AVAILABILITY STATEMENT}

Publicly available datasets were analyzed in this study. The datasets analyzed for this study can be obtained upon request from the Scientific Registry of Transplant Recipients (SRTR) [www.srtr.org].

\section{AUTHOR CONTRIBUTIONS}

$\mathrm{DB}, \mathrm{BM}, \mathrm{MR}$ and SS guided the research and wrote the manuscript. RG performed high-resolution HLA types imputation and re-matching simulation analyses. JBo and SK consulted and performed the statistical analyses. RL and AG

\section{REFERENCES}

1. Opelz G, Dohler B. Effect of human leukocyte antigen compatibility on kidney graft survival: Comparative analysis of two decades. Transplantation (2007) 84(2):137-43. doi: 10.1097/01.tp.0000269725.74189.b9

2. Williams RC, Opelz G, McGarvey CJ, Weil EJ, Chakkera HA. The Risk of Transplant Failure With HLA Mismatch in First Adult Kidney Allografts From Deceased Donors. Transplantation (2016) 100(5):1094-102. doi: 10.1097/TP.0000000000001115

3. Morris PJ, Johnson RJ, Fuggle SV, Belger MA, Briggs JD. Uktssa. Analysis of factors that affect outcome of primary cadaveric renal transplantation in the UK. Lancet (1999) 354(9185):1147-52. doi: 10.1016/S0140-6736(99)01104-6

4. Freitas MCS. Kidney transplantation in the US: an analysis of the OPTN/ UNOS registry. Clin Transpl (2011) 1-16.

5. Ashby VB, Port FK, Wolfe RA, Wynn JJ, Williams WW, Roberts JP, et al. Transplanting Kidneys Without Points for HLA-B Matching: Consequences of the Policy Change. Am J Transplant (2011) 11(8):1712-8. doi: 10.1111/ j.1600-6143.2011.03606.x

6. Nakamura T, Shirouzu T, Nakata K, Yoshimura N, Ushigome H. The Role of Major Histocompatibility Complex in Organ Transplantation- Donor Specific Anti-Major Histocompatibility Complex Antibodies Analysis Goes to the Next Stage. Int J Mol Sci (2019) 20(18):4544. doi: 10.3390/ ijms 20184544 performed 4-digit and 2-digit HLA identification in 1,095 patients, their statistical evaluation and HLA typing data analyses. BG, SL-M and JBr provided intellectual support for data processing and analyses. All authors contributed to the article and approved the submitted version.

\section{FUNDING}

Research reported in this publication was supported by the National Library of Medicine of the National Institutes of Health under Award Number R01LM013311 as part of the NSF/NLM Generalizable Data Science Methods for Biomedical Research Program. The content is solely the responsibility of the authors and does not necessarily represent the official views of the National Institutes of Health. Additional funding was provided by The Alliance for Paired Donation, Maumee, $\mathrm{OH}$, USA.

\section{ACKNOWLEDGMENTS}

The data reported here have been supplied by the Minneapolis Medical Research Foundation (MMRF) as the contractor for the Scientific Registry of Transplants Recipients (SRTR). The interpretation and reporting of these data are the responsibility of the author(s) and in no way should be seen as an official policy of or interpretation by the SRTR or the U.S. Government. Notably, the principles of the Helsinki Declaration were followed.

\section{SUPPLEMENTARY MATERIAL}

The Supplementary Material for this article can be found online at: https://www.frontiersin.org/articles/10.3389/fimmu.2020.580752/ full\#supplementary-material

7. Matas AJ, Smith JM, Skeans MA, Lamb KE, Gustafson SK, Samana CJ, et al. OPTN/SRTR 2011 Annual Data Report: Kidney. Am J Transplant (2013) 13:11-46. doi: 10.1111/ajt.12019

8. Lamb KE, Lodhi S, Meier-Kriesche HU. Long-Term Renal Allograft Survival in the United States: A Critical Reappraisal. Am J Transpl (2011) 11(3):45062. doi: 10.1111/j.1600-6143.2010.03283.x

9. Stegall MD, Stock PG, Andreoni K, Friedewald JJ, Leichtman AB. Why do we have the kidney allocation system we have today? A history of the 2014 kidney allocation system. Hum Immunol (2017) 78(1):4-8. doi: 10.1016/ j.humimm.2016.08.008

10. Organ Procurement and Transplantation Network (OPTN) Policies (2017).

11. Hart A, Smith JM, Skeans MA, Gustafson SK, Wilk AR, Robinson A, et al. OPTN/SRTR 2016 Annual Data Report: Kidney. Am J Transplant (2018) 18:18-113. doi: 10.1111/ajt.14557

12. Gebel HM, Kasiske BL, Gustafson SK, Pyke J, Shteyn E, Israni AK, et al. Allocating Deceased Donor Kidneys to Candidates with High Panel-Reactive Antibodies. Clin J Am Soc Nephrol (2016) 11(3):505-11. doi: 10.2215/CJN.07720715

13. Hart A, Smith JM, Skeans MA, Gustafson SK, Wilk AR, Castro S, et al. OPTN/ SRTR 2017 Annual Data Report: Kidney. Am J Transplant (2019) 19:19-123. doi: 10.1111/ajt.15274

14. Heidt S, Witvliet MD, Haasnoot GW, Claas FHJ. The 25th anniversary of the Eurotransplant Acceptable Mismatch program for highly sensitized patients. Transplant Immunol (2015) 33(2):51-7. doi: 10.1016/j.trim.2015.08.006 
15. Duquesnoy RJ. HLAMatchmaker: A molecularly based algorithm for histocompatibility determination. I. Description of the algorithm. Hum Immunol (2002) 63(5):339-52. doi: 10.1016/S0198-8859(02)00382-8

16. Kosmoliaptsis V, Chaudhry AN, Sharples LD, Halsall DJ, Dafforn TR, Bradley JA, et al. Predicting HLA Class I Alloantigen Immunogenicity From the Number and Physiochemical Properties of Amino Acid Polymorphisms. Transplantation (2009) 88(6):791-8. doi: 10.1097/TP.0b013e3181b4a9ff

17. Kosmoliaptsis V, Sharples LD, Chaudhry AN, Halsall DJ, Bradley JA, Taylor CJ. Predicting HLA Class II Alloantigen Immunogenicity From the Number and Physiochemical Properties of Amino Acid Polymorphisms. Transplantation (2011) 91(2):183-90. doi: 10.1097/TP.0b013e3181ffff99

18. Kosmoliaptsis V, Mallon DH, Chen Y, Bolton EM, Bradley JA, Taylor CJ. Alloantibody Responses After Renal Transplant Failure Can Be Better Predicted by Donor-Recipient HLA Amino Acid Sequence and Physicochemical Disparities Than Conventional HLA Matching. Am J Transpl (2016) 16(7):2139-47. doi: 10.1111/ajt.13707

19. Wiebe C, Kosmoliaptsis V, Pochinco D, Taylor CJ, Nickerson P. A Comparison of HLA Molecular Mismatch Methods to Determine HLA Immunogenicity. Transplantation (2018) 102(8):1338-43. doi: 10.1097/ TP. 0000000000002117

20. Kosmoliaptsis V, Joris MM, Mallon DH, Lankester AC, von dem Borne PA, Kuball J, et al. Physiochemical disparity of mismatched HLA class I alloantigens and risk of acute GVHD following HSCT. Bone Marrow Transplant (2015) 50(4):540-4. doi: 10.1038/bmt.2014.305

21. Mallon DH, Kling C, Robb M, Ellinghaus E, Bradley JA, Taylor CJ, et al. Predicting Humoral Alloimmunity from Differences in Donor and Recipient HLA Surface Electrostatic Potential. J Immunol (2018) 201(12):3780-92. doi: 10.4049/jimmunol.1800683

22. Duquesnoy RJ. Are We Ready for Epitope-Based HLA Matching in Clinical Organ Transplantation? Transplantation (2017) 101(8):1755-65. doi: 10.1097/TP.0000000000001667

23. De Santis D, Truong L, Martinez P, D’Orsogna L. Rapid high-resolution HLA genotyping by MinION Oxford nanopore sequencing for deceased donor organ allocation. Hla (2020) 96(2):141-62. doi: 10.1111/tan.13901

24. Maiers M, Gragert L, Klitz W. High-resolution HLA alleles and haplotypes in the United States population. Hum Immunol (2007) 68(9):779-88. doi: 10.1016/j.humimm.2007.04.005

25. Holdsworth R, Hurley CK, Marsh SGE, Lau M, Noreen HJ, Kempenich JH, et al. The HLA dictionary 2008: a summary of HLA-A, -B, -C,-DRB1/3/4/5, and-DQB1 alleles and their association with serologically defined HLA-A, -B, -C, -DR, and -DQ antigens. Tissue Antigens (2009) 73(2):95-170. doi: 10.1111/j.1399-0039.2008.01183.x

26. Madbouly A, Gragert L, Freeman J, Leahy N, Gourraud PA, Hollenbach JA, et al. Validation of statistical imputation of allele-level multilocus phased genotypes from ambiguous HLA assignments. Tissue Antigens (2014) 84 (3):285-92. doi: 10.1111/tan.12390

27. Neville MJ, Lee W, Humburg P, Wong D, Barnardo M, Karpe F, et al. High resolution HLA haplotyping by imputation for a British population bioresource. Hum Immunol (2017) 78(3):242-51. doi: 10.1016/ j.humimm.2017.01.006

28. Milner J, Melcher ML, Lee B, Veale J, Ronin M, D’Alessandro T, et al. HLA Matching Trumps Donor Age: Donor-Recipient Pairing Characteristics That Impact Long-Term Success in Living Donor Kidney Transplantation in the Era of Paired Kidney Exchange. Transplant Direct (2016) 2(7):1-17 e85. doi: 10.1097/TXD.0000000000000597

29. Brazier F, Jouffroy J, Martinez F, Thao NK, Anglicheau D, Legendre C, et al. Association of blood bicarbonate and $\mathrm{pH}$ with mineral metabolism disturbance and outcome after kidney transplantation. Am J Transplant (2020) 20(4):1063-75. doi: 10.1111/ajt.15686

30. Bursac Z, Gauss CH, Williams DK, Hosmer DW. Purposeful selection of variables in logistic regression. Source Code Biol Med (2008) 3:17. doi: 10.1186/1751-0473-3-17

31. Inc. SI. SAS/STAT(R) 9.2 User"s Guide, Second Edition Cary, NC: SAS Institute Inc. (2009). Available at: https://support.sas.com/documentation/cdl/en/ statug/63033/HTML/default/viewer.htm\#statug_phreg_sect007.htm.

32. Lin DY, Wei LJ, Ying Z. Checking the Cox Model with Cumulative Sums of Martingale-Based Residuals. Biometrika (1993) 80(3):557-72. doi: 10.1093/ biomet/80.3.557
33. Borucka J. Extensions of Cox Model for Non-Proportional Hazards Purpose. Econometrics (2014) 3(45):1-10. doi: 10.15611/ekt.2014.3.07

34. Moen T, Albrechtsen D, Flatmark A, Jakobsen A, Jervell J, Halvorsen S, et al. IMPORTANCE OF HLA-DR MATCHING IN CADAVERIC RENALTRANSPLANTATION - A PROSPECTIVE ONE-CENTER STUDY OF 170 TRANSPLANTS. New Engl J Med (1980) 303(15):850-4. doi: 10.1056/ NEJM198010093031504

35. Coupel S, Giral-Classe M, Karam G, Morcet JF, Dantal J, Cantarovich D, et al. Ten-year survival of second kidney transplants: Impact of immunologic factors and renal function at 12 months. Kidney Int (2003) 64(2):674-80. doi: 10.1046/j.1523-1755.2003.00104.x

36. Tambur AR, Leventhal JR, Zitzner JR, Walsh RC, Friedewald JJ. The DQ Barrier: Improving Organ Allocation Equity Using HLA-DQ Information. Transplantation (2013) 95(4):635-40. doi: 10.1097/TP.0b013e318277b30b

37. OPTN. Eliminate the Use of DSA and Region from Kidney Allocation Policy. In: Network OPaT. U.S. Department of Health and Human Services (2019).

38. Ashby VB, Leichtman AB, Rees MA, Song PXK, Bray M, Wang W, et al. A Kidney Graft Survival Calculator that Accounts for Mismatches in Age, Sex, HLA, and Body Size. Clin J Am Soc Nephrol (2017) 12(7):1148-60. doi: 10.2215/CJN.09330916

39. Manski CF, Tambur AR, Gmeiner M. Predicting kidney transplant outcomes with partial knowledge of HLA mismatch. Proc Natl Acad Sci U States America (2019) 116:20339-45. doi: 10.1073/pnas.1911281116

40. Unterrainer C, Dohler B, Susal C. Pitfalls in graft survival analysis. Hla (2018) 91(2):80-7. doi: 10.1111/tan.13191

41. McCaughan JA, Courtney AE. The Clinical Course of Kidney Transplant Recipients After 20 Years of Graft Function. Am J Transpl (2015) 15(3):73440. doi: 10.1111/ajt.13041

42. Vincenti F. A decade of progress in kidney transplantation. Transplantation (2004) 77(9):S52-61. doi: 10.1097/01.TP.0000126928.15055.DC

43. Wagner SJ, Brennan DC. Induction Therapy in Renal Transplant Recipients How Convincing is the Current Evidence? Drugs (2012) 72(5):671-83. doi: 10.2165/11631300-000000000-00000

44. Lachmann N, Terasaki PI, Budde K, Liefeldt L, Kahl A, Reinke P, et al. AntiHuman Leukocyte Antigen and Donor-Specific Antibodies Detected by Luminex Posttransplant Serve as Biomarkers for Chronic Rejection of Renal Allografts. Transplantation (2009) 87(10):1505-13. doi: 10.1097/TP.0b013e3181a44206

45. Terasaki PI. Humoral theory of transplantation. Am J Transplant (2003) 3 (6):665-73. doi: 10.1034/j.1600-6143.2003.00135.x

46. Sellares J, Reeve J, Loupy A, Mengel M, Sis B, Skene A, et al. Molecular Diagnosis of Antibody-Mediated Rejection in Human Kidney Transplants. Am J Transplant (2013) 13(4):971-83. doi: 10.1111/ajt.12150

47. Duquesnoy RJ. Reflections on HLA Epitope-Based Matching for Transplantation. Front Immunol (2016) 7:469. doi: 10.3389/fimmu.2016.00469

48. Philogene MC, Amin A, Zhou S, Charnaya O, Vega R, Desai N, et al. Eplet mismatch analysis and allograft outcome across racially diverse groups in a pediatric transplant cohort: a single-center analysis. Pediatr Nephrol (2020) 35 (1):83-94. doi: 10.1007/s00467-019-04344-1

49. Sapir-Pichhadze R, Zhang X, Ferradji A, Madbouly A, Tinckam KJ, Gebel $\mathrm{HM}$, et al. Epitopes as characterized by antibody-verified eplet mismatches determine risk of kidney transplant loss. Kidney Int (2020) 97(4):778-85. doi: 10.1016/j.kint.2019.10.028

50. Kramer CSM, Israeli M, Mulder A, Doxiadis IIN, Haasnoot GW, Heidt S, et al. The long and winding road towards epitope matching in clinical transplantation. Transplant Int (2019) 32(1):16-24. doi: 10.1111/tri.13362

\section{Conflict of Interest: JB was employed by Parexel International.}

The authors declare that the research was conducted in the absence of any commercial or financial relationships that could be construed as a potential conflict of interest.

Copyright (c) 2020 Bekbolsynov, Mierzejewska, Borucka, Liwski, Greenshields, Breidenbach, Gehring, Leonard-Murali, Khuder, Rees, Green and Stepkowski. This is an open-access article distributed under the terms of the Creative Commons Attribution License (CC BY). The use, distribution or reproduction in other forums is permitted, provided the original author(s) and the copyright owner(s) are credited and that the original publication in this journal is cited, in accordance with accepted academic practice. No use, distribution or reproduction is permitted which does not comply with these terms. 